\title{
National Proficiency Testing of Molecular Diagnostics for Tuberculosis and Drug Resistance Detection - China, 2014-2019
}

\author{
Xichao Ou'; Hui Xia'; Shengfen Wang'; Bing Zhao'; Jiale Fan'; Yuanyuan Song'; \\ Yang Zhou'; Yang Zheng'; Yanlin Zhao ${ }^{1, *}$
}

\section{Summary}

What is already known about this topic?

Proficiency testing (PT) is a key component of quality assurance and is essential in ensuring accurate laboratory diagnosis of tuberculosis (TB) and drugresistant TB. The National Tuberculosis Reference Laboratory (NTRL) developed a novel PT panel to test laboratories' proficiency for molecular TB diagnostic assays throughout the TB laboratory network. A total of 6 PT rounds for molecular diagnostics were conducted by NTRL from 2014 to 2019.

\section{What is added by this report?}

PT conducted using artificial sputum specimens increased from 120 in the first round to 1,835 in the sixth round. Overall, laboratories demonstrated good proficiency for MTB and drug-resistance detection by molecular diagnostics, which is evident from the qualification rates over the six rounds: $95 \%, 97 \%$, $96 \%, 93 \%, 93 \%$, and $97 \%$, respectively.

What are the implications for public health practice?

The use of artificial sputum specimens for PT panel production to test TB molecular diagnostics in China is feasible. Most of the participating laboratories provided reliable molecular diagnostic results for MTB and drugresistance detection. The TB laboratory network can be instrumental in implementing PT expansion and improving the quality of $\mathrm{TB}$ molecular diagnosis in China.

China has made significant progress in establishing a well-coordinated tuberculosis (TB) laboratory network (1). The national TB laboratory network is composed of four levels of laboratories: national, provincial, prefectural, and county. The National TB Reference Laboratory (NTRL) is responsible for the development of the national plan for TB lab services and provides training and guidance to lower-level TB labs. NTRL leads the provincial-level TB reference laboratory (PTRL) plan and oversees the services provided by prefectural and county-level TB labs to ensure quality and compliance with biosafety regulations.

Over the past few years, several new commercial molecular diagnostics have been recommended by the World Health Organization (WHO) (2-4). In addition, several locally manufactured molecular diagnostics were certified for use by China's National Medical Products Administration (NMPA) (5-7). Following this, in 2013, the Global Fund procured 925 GeneXpert and 125 GenoType MTBDRplus instruments for China along with test reagents. China's "13th Five-Year" National Tuberculosis Prevention and Control Plan (for implementation by 2020) mandated that all TB-designated hospitals at the prefecture level shall have the ability to carry out molecular diagnosis of $\mathrm{TB}, 80 \%$ of counties in the eastern and central regions and $70 \%$ of counties in the western regions shall have the capacity to carry out molecular diagnosis of tuberculosis. With past and future rapid scale up of TB molecular diagnostics across the country, there has been and remains an urgent need for a proficiency testing (PT) program to ensure the quality of these new testing platforms (8). PT for TB molecular diagnostics was first launched in 2014, and as of 2019, six rounds have been completed. This was the first study in China that systematically assessed the procedures and results of the nationwide proficiency testing of molecular diagnostic tools.

Each PT panel contained 10 artificial sputum specimens with the following characteristics: 2 rifampin (RIF) and isoniazid (INH)-susceptible mycobacterium TB complex (MTBC), 2 RIF and INH-resistant MTBC, 2 RIF-resistant MTBC, 1 INH-resistant MTBC, 1 non-TB mycobacterium (NTM), and 2 negative for TB. All PT panels were transported from the NTRL to targeted provincial TB reference laboratories (PTRL), and each of these 
provincial sites were then arranged for distribution of panels to prefecture and county-level TB laboratories. All transport and storage of panels from the NTRL to the final testing sites was done with strict observance of cold chain.

All laboratories capable of molecular diagnosis of TB were required to participate in the PT plan. Once testing of PT panels was completed, all PTRL were responsible for collection and submission of test results (from participating laboratories) to NTRL. The NTRL compared the submitted test results with the reference results for each participating laboratory. Laboratory sites passed PT and determined as qualification if their results had no errors in the qualitative diagnosis of MTB, rifampicin, or isoniazid resistance or only had one sample with no test result (i.e. test failure). NTRL issued certificates acknowledging the type of molecular diagnostic platform used, provided results feedback, and discussed findings with the staff of all participating laboratories.

The number of participating laboratories from the first to sixth rounds of PT increased each year. Laboratory participation increased from 120 in the first round to 1,835 in the sixth round. Laboratories participating in the first round of PT were predominantly provincial and prefectural-level facilities and had a qualification rate of $95 \%$. The second round of testing technology is GeneXpert with a qualification rate of $97 \%$. With the increase in the number of county-level testing labs and the types of diagnostics, the qualification rates of the fourth and fifth rounds dropped to $93 \%$. After national and provincial-level training programs were strengthened, the sixth-round qualification rate increased to $97 \%$ (Figure 1).

Except for the first round of testing, which did not include county-level TB labs, all other rounds of PT were primarily performed at county-level TB labs, which accounted for $72 \%(966 / 1,341)$ and $75 \%$ $(1,370 / 1,835)$ at the fifth and sixth round PT, and 4 third-party medical testing centers joined in the sixth round (Figure 2).

The type of participating facilities transitioned from CDCs and TB dispensaries in the first four rounds to TB-designated hospitals in the fifth and sixth rounds. TB-designated hospitals accounted for $65 \%$ $(1,199 / 1,835)$ in the sixth round (Figure 2$)$.

A total of 1,971 sets of PT panels were tested by a variety of molecular diagnostic platforms in the sixth round PT plan, and the qualification rate was $96.3 \%$ $(1,899 / 1,971)$. Of all participating facilities, most were county-level facilities, accounting for $75 \%$ $(1,370 / 1,835)$. The qualification rates at the provincial, prefectural, and county-levels were $97 \%$ (69/71), 94\% (366/390), and 97\% (1,331/1,370), respectively. The qualification rates of TB-designated hospitals and $\mathrm{CDC} / \mathrm{TB}$ dispensary were $97 \%$ $(1,155 / 1,196)$ and $96 \%(610 / 633)$, respectively.

All the provincial labs carried out Xpert MTB/RIF testing in the six round PT, three isothermal amplification diagnostics were covered in a total of 20 provincial-level administrative divisions (PLADs). Except Xizang (Tibet), all provincial labs have molecular drug sensitivity testing capabilities. Xpert MTB/RIF (48\%, 953/1,971) was the most predominant molecular method, followed by Defast.TB (14\%, 285/1,971), EasyNat (13\%, 255/1,971), MeltPro TB (7\%, 144/1,971), real-time PCR $(5 \%, 100 / 1,971)$, GenoType MTBDRplus $(5 \%$, 98/1,971), Loopamp (4\%, 74/1,971), and GeneChip $\operatorname{MDR} \operatorname{TB}(3 \%, 62 / 1,971)$.

Different molecular diagnostic tools had different qualification rates in the sixth PT plan. EasyNat has

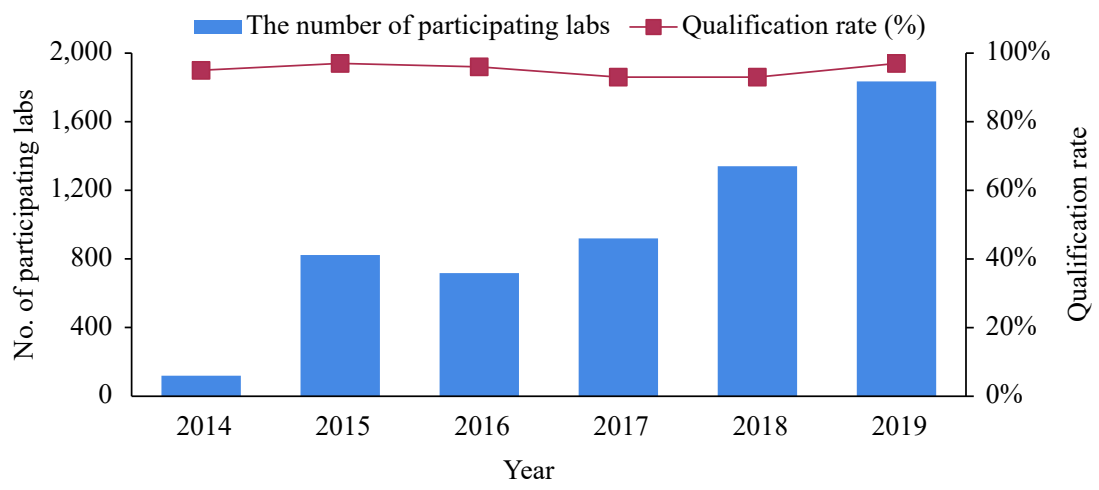

FIGURE 1. Number of participating labs and qualification rate for proficiency testing.

Note: The data on the left axis represents the number of participating labs. The percentage on the right axis represents the qualification rate of participating labs. 
A $₫$ Provincial $\backsim$ Prefectural $\llbracket$ County $₫$ Other
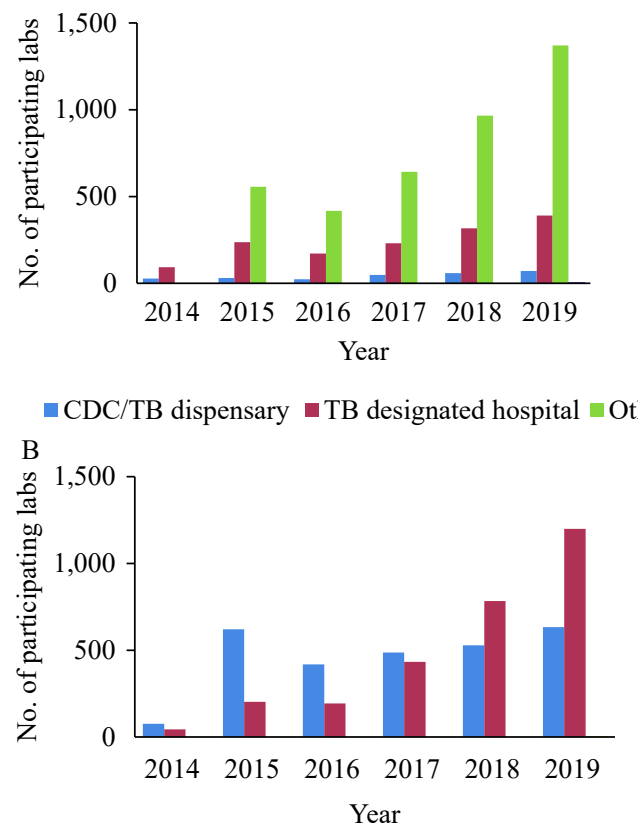

FIGURE 2. Levels and types of participating units in proficiency testing.

Note: The data on the left axis represents the number of participating labs.

the lowest qualification rates at $92 \%$ (234/255), with error types being mainly false negative for MTB detection and appeared more than once in some labs. Xpert MTB/RIF had the highest qualification rates at $98 \%(931 / 953)$.

The false positive rate of unqualified MTB detection was $16 \%$ (19/119), while the false negative rate of unqualified MTB detection was 63\% (75/119). A small number of laboratories encountered problems with identification of non-tuberculous mycobacteria (NTM) and drug susceptibility testing to RFP and INH, 7 samples were misdiagnosed as MTB, and 10 samples of RFP and 7 samples of INH susceptibility test results were incorrect (Table 1).

\section{DISCUSSION}

Quality assurance is intended to ensure the quality of the overall testing process (8). The NTRL provides quality assurance through technical, biosafety, and managerial training; proficiency testing; and onsite supervisory. The aim of this study was to systematically analyze the performance of $\mathrm{TB}$ laboratories for detecting MTB and drug susceptibility through a limited series of PT rounds. This evaluation identified common problems at the laboratory level, assessed staff

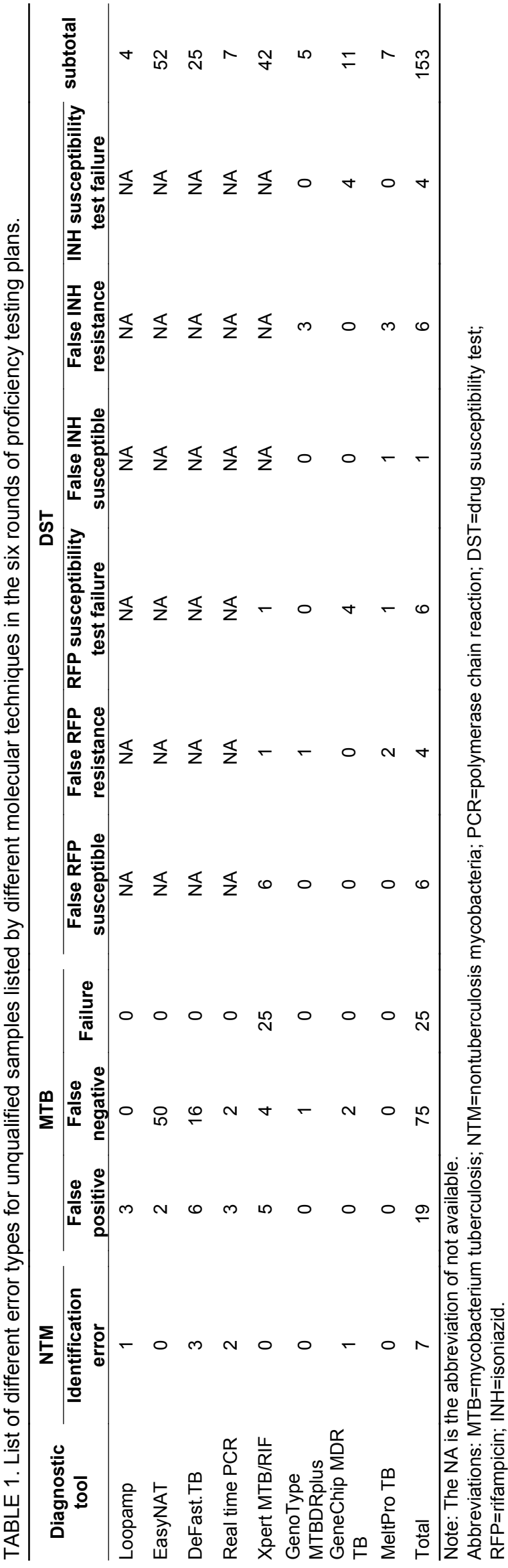


and management training needs, and highlighted the potential benefits of a long-term, permanent PT plan for all TB diagnostic testing sites in China.

This study identified that participation rates in PT plans were relatively low at first, which was likely due to not all laboratories performing molecular diagnostics routinely and the NTRL just carrying out PT for Global Fund project sites that had already been equipped with GenoType MTBDR and GeneXpert equipment. The 13th Five-Year National Tuberculosis Prevention and Control Plan stipulated that each PLAD initiate molecular testing for TB detection and provide financial support for participation in PT plans, which all contributed to the successful implementation of the fifth and sixth rounds of PT plans. This is an example of the value of national policy working handin-hand with national public health programs to improve quality of diagnostic testing.

Overall, the participating laboratories demonstrated good performance during the PT plan. Contamination was the most serious concern for clinical laboratories' use of molecular diagnostics. To avoid contamination during panel sample preparation, all of the PT samples were prepared by anti-contamination operations in a biosafety-level III laboratory. The false positive rate for unqualified MTB detection was $16 \%$ (19/119) in the sixth round of PT. Five negative samples were read as positive by Xpert MTB/RIF, which is a reminder that even with fully automated integrated testing equipment, test personnel still need to strictly adhere to the standardized operating procedures and should regularly clean and maintain testing equipment to avoid contamination (9).

In this study, we also found that some positive PT samples produced negative results, which could have been due to the following reasons: failure of nucleic acid extraction, instrument failure, or problems with test reagents. Once again, this illustrates the need for laboratory staff to do the following: 1) strictly follow the standardized operating procedures for nucleic acid extraction; 2) strictly follow the kit manufacturer's instructions and requirements for reagent transportation and storage; 3) regularly check and calibrate the consistency of the temperature control of the thermal cycler; and 4) speed up the development and application of automated testing equipment for nucleic acid extraction, amplification, and detection.

Some other problems seen in individual laboratories included clerical errors, incorrect interpretation of test results, and test failure issues. These problems may be indicative of more general problems in laboratory practice and should be corrected by implementing a laboratory quality management system. Corrective actions concerning interdependent quality management systems activities could include the following: conducting staff training and refresher training in case of operator-related non-conformities; maintaining and updating registers; monitoring quality indicators; reviewing sample referral systems; and improving and updating the infrastructure of laboratory facilities. In addition, laboratory practices may be subject to the Hawthorne Effect, in which site performance improved due to regular quality assessment, so that PT should be combined with technical training and onsite supervisory to perform better quality assurance.

This study was subject to two limitations. First, not all laboratories capable of molecular diagnosis of TB had participated in the PT plan. With more policies and strategies having been enforced, this situation has been changed greatly in the sixth round PT plan. Second, the cold chain requirement is likely to be a risk and cost issue in our PT plan, but the liquid specimen we used had resemblance to clinical samples and were relatively cost-effective to produce. We overcame this difficulty to some extent by tapping into the national TB laboratory network.

In conclusion, this study suggests that it is possible to develop a PT program (for TB molecular diagnostics) in a country as large and populous as China and that the use of artificial sputum specimens was feasible for this purpose. This implementation study demonstrated that the participating laboratories showed good performance in all six PT rounds and TB laboratory networks can be instrumental in implementing PT for expanding and improving TB molecular diagnosis in China.

Funding: National Science and Technology Major Project (2018ZX10103001).

Conflicts of Interest: The authors report no conflicts of interest related to this article.

Acknowledgments: Mr. Kyle Bond (United States CDC) and Dr. Ling Hao (United States CDC China Office).

doi: $10.46234 / \mathrm{ccdcw} 2021.072$

\# Corresponding author: Yanlin Zhao, zhaoyl@chinacdc.cn.

\footnotetext{
${ }^{1}$ National Center for Tuberculosis Control and Prevention, Chinese Center for Disease Control and Prevention, Beijing, China.
}

Submitted: February 18, 2021; Accepted: March 18, 2021 


\section{REFERENCES}

1. Zhao YL. Quality assurance manual for TB laboratory. Beijing: People's Medical Publishing House. 2017. (In Chinese).

2. World Health Organization. Xpert MTB/RIF assay for the diagnosis of pulmonary and extrapulmonary TB in adults and children: WHO Policy update. Geneva: World Health Organization. 2014. https://www.who. int/tb/publications/xpert_policyupdate/en/. [2021-02-16].

3. World Health Organization. The use of molecular line probe assays for the detection of resistance to isoniazid and rifampicin. Geneva: World Health Organization. 2016. https://www.who.int/tb/publications/molecu lar-test-resistance/en/. [2021-02-16].

4. World Health Organization. The use of loop-mediated isothermal amplification (TB-LAMP) for the diagnosis of pulmonary tuberculosis: policy guidance. Geneva: World Health Organization. 2016. https:// www.who.int/tb/publications/lamp-diagnosis-molecular/en/. [2021-0216].

5. Ou XC, Wang SF, Dong HY, Pang Y, Li Q, Xia H, et al. Multicenter evaluation of a real-time loop-mediated isothermal amplification (RealAmp) test for rapid diagnosis of Mycobacterium tuberculosis. J Microbiol Methods 2016;129:39 - 43. http://dx.doi.org/10.1016/j. mimet.2016.07.008.

6. Guo Y, Zhou Y, Wang C, Zhu L, Wang S, Li Q, et al. Rapid, accurate determination of multidrug resistance in $M$. tuberculosis isolates and sputum using a biochip system. Int J Tuberc Lung Dis 2009;13(7): 914 20. https://pubmed.ncbi.nlm.nih.gov/19555544/.

7. Ou XC, Song YY, Zhao B, Li Q, Xia H, Zhou Y, et al. A multicenter study of cross-priming amplification for tuberculosis diagnosis a peripheral level in China. Tuberculosis 2014;94(4):428 - 33. http://dx. doi.org/10.1016/j.tube.2014.04.006).

8. Noble MA. Does external evaluation of laboratories improve patient safety? Clin Chem Lab Med 2007;45(6):735 - 5. http://dx.doi.org/10 1515/CCLM.2007.166.

9. Scott LE, Gous N, Cunningham BE, Kana BD, Perovic O, Erasmus L, et al. Dried culture spots for Xpert MTB/RIF external quality assessment: results of a phase 1 pilot study in South Africa. J Clin Microbiol 2011;49(12):4356 - 60. http://dx.doi.org/10.1128/JCM.05167-11. 